\title{
Rôle des herpèsvirus humains 6 (HHV-6) dans la prédisposition à la pré-éclampsie
}

\author{
Pascale Bonnafous ${ }^{1}$, Agnès Gautheret-Dejean ${ }^{2-4 *}$
}

\section{Diversité des formes d'herpèsvirus} humain 6 (HHV-6)

Au sein de la famille des herpèsvirus humains, le ou plutôt les HHV-6 ont fait l'objet de recherches récentes, qui ont permis de mieux comprendre les mécanismes de l'infection par ces virus et leur rôle dans diverses maladies. Le génome des HHV-6 est constitué d'un ADN double-brin linéaire qui comporte une séquence unique contenant une centaine de gènes (Ul à U100), et des séquences terminales orientées $D R R$ et $D R L$ (direct repeat right and left). Les deux variants de ce virus initialement identifiés ont été classés en deux espèces, HHV-6A et HHV-6B, du fait de caractéristiques génomiques, antigéniques, de tropisme et de pathogénicité distinctes. Ce sont des virus opportunistes, qui persistent dans l'organisme à l'état latent dans quelques types cellulaires, sous forme épisomale ou intégrée dans le télomère d'un chromosome: en effet, la présence, aux extrémités du génome viral, de séquences répétées identiques aux séquences télomériques humaines, favorise une recombinaison avec celles-ci. La réactivation de ces virus, fréquente, est asymptomatique chez les sujets immunocompétents, mais peut provoquer diverses maladies chez les individus immunodéprimés: fièvre avec rash cutané et cytopénie, encéphalite, hépatite, retard à la sortie d'aplasie, syndrome d'hypersensibilité médicamenteuse, etc. La séroprévalence des HHV-6 est supérieure à $90 \%$ dans la population des adultes, et on estime que 90 à $95 \%$ des réactivations observées sont dues à HHV-6B. En dehors de l'Afrique, les infections par HHV-6A semblent beaucoup plus rares et acquises plus tardivement.

Par ailleurs, environ 1 individu sur 100 est porteur d'une forme de HHV-6A ou HHV-6B intégrée dans I'ADN des cellules germinales, qui est alors transmise de façon héréditaire selon un mode mendélien (inherited chromosomally integrated HHV-6, iciHHV-6) [1] $(\rightarrow)$.

Cette propor-

$\rightarrow$ Voir la Nouvelle de A. GautheretDejean, $m / s n^{\circ} 8-9$, août-septembre 2017, page 730

tion d'individus porteurs d'iciHHV-6 varie légèrement selon les régions $d u$ monde: de 0 à $0,3 \%$ pour iciHHV-6A et de 0 à 2,8\% (maximum observé au nord du Royaume-Uni) pour iciHHV-6B, d'après les données épidémiologiques actuelles. L'espèce HHV-6B représente donc la majorité des formes intégrées de HHV-6 : environ $55 \%$ des cas dans de nombreux pays, dont la France, et jusqu'à 100 \% dans le nord du RoyaumeUni. Chez ces individus, la présence du virus dans toutes les cellules de l'organisme est responsable, quel que soit le prélèvement, de la détection d'une forte charge virale, qui peut être interprétée à tort comme le signe d'une infection très active. La détection du virus dans les follicules pileux ou les ongles permet de le distinguer d'une souche HHV-6A ou HHV-6B classique, qui n'infecte jamais ces tissus. Toutefois, il a été prouvé qu'un iciHHV-6 pouvait également se réactiver, avec de graves conséquences médicales [1, 2]. Le diagnostic est alors plus compliqué, puisque la charge virale détectée est identique à celle d'un iciHHV-6 latent.
ISorbonne Université, Inserm U1136, Institut Pierre Louis d'épidémiologie et de santé publique (IPLESP), Faculté de médecine, site Pitié-Salpêtrière, service de virologie, bâtiment CERVI, 83 boulevard de l'Hôpital, 75013 Paris, France.

${ }^{2}$ AP-HP, Hôpitaux universitaires La Pitié Salpêtrière-Charles Foix, service de virologie, bâtiment CERVI, 83 boulevard de l'Hôpital

75013 Paris, France.

3 Université de Paris, Inserm UMR-S U1139 3PHM, 4 avenue de l'Observatoire, 75006 Paris, France.

${ }^{4}$ Université de Paris, Faculté de pharmacie de Paris, laboratoire de microbiologie, 4 avenue de l'Observatoire, 75006 Paris, France.

*Agnès Gautheret-Dejean est coordinatrice du CNR-Laboratoire associé du CNR Herpèsvirus pour l'étude du HHV-6. pascale.bonnafous@sorbonne-universite.fr agnes.gautheret@aphp.fr

Une pathogénicité des iciHHV-6 encore mal connue

Si les individus porteurs de iciHHV-6 ne semblent pas présenter de symptômes spécifiques, l'étude d'une grande cohorte d'individus vivant au Canada a toutefois montré qu'ils avaient un risque accru (environ triple) d'angine de poitrine [3]. Chez des patients recevant une transplantation de cellules souches hématopoïétiques, un risque augmenté de réaction du greffon contre l'hôte et de virémie à cytomégalovirus a été mis en évidence [4]. Concernant l'infection congénitale, il a été montré que, hormis la transmission génétique mendélienne représentant $86 \%$ des cas, les $14 \%$ de cas restants avaient pour origine un iciHHV-6 maternel et non un HHV-6 opportuniste. Dans ces cas, la réactivation de la forme intégrée chez la mère, avec production de particules virales et passage transplacentaire, entraîne l'infection du fœtus [5]. On retrouve ainsi un HHV-6 de la même espèce que le iciHHV-6 maternel dans le sang du cordon ombilical, puis, éventuellement, dans le sang périphérique et dans la salive de l'enfant. Les conséquences sur son développement, en 
particulier neurologique, sont encore à l'étude. Il est important de noter que les études publiées n'ont pas toujours distingué iciHHV-6A et iciHHV-6B, et que les manifestations cliniques pourraient être différentes selon l'espèce virale impliquée.

\section{Étude de la prévalence de l'infection} par HHV-6 dans la pré-éclampsie ${ }^{1}$

Une étude britannique s'est penchée sur les mécanismes physiopathologiques de la pré-éclampsie, et les auteurs ont émis l'hypothèse d'une infection virale [6]. Par une technique de séquençage à haut-débit, ils ont analysé le transcriptome dans des biopsies du placenta effectuées au moment de l'accouchement chez des femmes présentant une pré-éclampsie (99 cas), un retard de la croissance du fœtus (48 cas), ou dont la grossesse s'est déroulée normalement (témoins, 132 cas). Les seuls ARNm non humains retrouvés étaient ceux des HHV-6A et HHV-6B, présents dans les trois groupes de femmes, plus fréquemment détectés dans le groupe des femmes présentant une pré-éclampsie (6\%) que dans les deux autres groupes réunis (2\%).

Une identification d'espèce et une quantification par amplification génique quantitative (quantitative polymerase chain reaction, $\mathrm{qPCR}$ ) dans le placenta ainsi que dans des prélèvements de sang maternel et de salive paternelle ont permis de déterminer qu'il s'agissait d'iciHHV-6, d'origine maternelle ou paternelle, pour plusieurs enfants (Tableau 1). L'identité des séquences virales placentaire et parentale a pu être prouvée grâce au polymorphisme de certaines positions nucléotidiques (single nucleotide polymorphism, SNP), au nombre de 999 pour HHV-6A et de

\footnotetext{
1 La pré-éclampsie, appelée aussi toxémie gravidique, est une hypertension artérielle, associée à une protéinurie, qui apparaît dans la deuxième moitié de la grossesse. Elle affecte de $2 \%$ à $8 \%$ des grossesses, selon les pays et l'ethnie. Lorsqu'elle n'est pas détectée à temps, voire prévenue par un traitement approprié dans les grossesses à risque, elle peut évoluer vers l'éclampsie, qui se manifeste par des convulsions et constitue une situation d'urgence vitale.
}

\begin{tabular}{|c|c|c|c|c|c|}
\hline Enfant & $\begin{array}{l}\text { Groupe } \\
\text { d'étude }\end{array}$ & $\begin{array}{c}\text { Espèce } \\
\text { de } \\
\text { HHV-6 }\end{array}$ & $\begin{array}{c}\text { Origine } \\
\text { de } \\
\text { iciHHV-6 }\end{array}$ & $\begin{array}{l}\text { Nombre de } \\
\text { fragments } \\
\text { de transcrits }\end{array}$ & $\begin{array}{c}\text { Répartition } \\
\text { de ces } \\
\text { fragments }\end{array}$ \\
\hline 1 & $T$ & $\begin{array}{c}\text { iciHHV- } \\
6 \mathrm{~B}\end{array}$ & maternelle & 331 & génome entier \\
\hline 2 & $T$ & $\begin{array}{l}\text { iciHHV- } \\
6 \mathrm{~B}\end{array}$ & paternelle & 259 & $\begin{array}{l}\text { génome entier } \\
\qquad \text { (U100 } \\
\text { prédominant) }\end{array}$ \\
\hline 3 & RCIU & $\begin{array}{c}\text { iciHHV- } \\
6 \mathrm{~A}\end{array}$ & maternelle & 77 & génome entier \\
\hline 4 & $T$ & $\begin{array}{c}\text { iciHHV- } \\
6 \mathrm{~B}\end{array}$ & paternelle & 43 & U58 à U100, DRR \\
\hline 5 & $P \varepsilon$ & HHV-6A & - & 22 & $D R L$ et $D R R$ \\
\hline 6 & $P \varepsilon$ & $\begin{array}{l}\text { iciHHV- } \\
6 \mathrm{~B}\end{array}$ & paternelle & 9 & $\begin{array}{c}\text { U17, U28, U31, } \\
\text { U76 }\end{array}$ \\
\hline 7 & $P \varepsilon$ & $\begin{array}{c}\text { iciHHV- } \\
6 \mathrm{~B}\end{array}$ & maternelle & 7 & $\begin{array}{l}\text { U50, U57, U90, } \\
\text { U95, R3 }\end{array}$ \\
\hline 8 & $P \varepsilon$ & $\begin{array}{c}\text { iciHHV- } \\
6 \mathrm{~A}\end{array}$ & paternelle & 3 & U60, U94 \\
\hline 9 & $P \varepsilon$ & HHV-6A & - & 1 & U78 \\
\hline 10 & $P \varepsilon$ & HHV-6B & - & 1 & U94 \\
\hline
\end{tabular}

Tableau I. Expression (transcriptome) et origine des souches de HHV-6 détectées dans les biopsies de placenta. T : témoin; RCIU : retard de croissance intra-utérin; PE : pré-éclampsie.

187 pour HHV-6B. Pour l'un des couples enfant-parent avec un iciHHV-6B, le site télomérique d'intégration a même été déterminé par séquençage. L'étude d'une autre cohorte (5061 prélèvements de sang de cordon) a confirmé la réactivation fréquente des iciHHV-6 : les fœtus porteurs d'iciHHV-6 ont été définis par la présence d'au moins une copie d'ADN viral (quantification des gènes U67/68) par cellule (quantification du gène codant la ribonucléase $P$ RNA component $H 1, \mathrm{RPPH}$ ), et le transcrit viral U100 a été détecté (par RT-qPCR) chez $69 \%$ d'entre eux.

Chez les trois autres enfants du groupe des femmes présentant une pré-éclampsie et non porteurs de iciHHV-6, les ARNm détectés pourraient résulter de la réactivation d'une souche maternelle de HHV-6A ou de HHV-6B. Dans un cas où seules les régions $D R_{L} / R$ étaient exprimées, l'hypothèse de l'intégration d'une forme virale tronquée de sa région unique, comme cela a été décrit précédemment [7], n'a toutefois pas été envisagée par les auteurs. De plus, la mise en évidence d'un faible taux d'expression, avec moins de transcrits viraux détectés dans le groupe des femmes présentant une pré-éclampsie que dans le groupe témoin, pose question. Les auteurs font remarquer que l'étude du transcriptome a été faite uniquement sur une portion du placenta après l'accouchement, et que l'expression des gènes viraux pourrait être différente pour l'ensemble du placenta au cours de la grossesse. Si le niveau d'expression est variable, la prévalence de iciHHV-6 chez le fœtus est néanmoins plus élevée dans le groupe des femmes présentant une pré-éclampsie que chez les témoins et a été confirmée dans d'autres cohortes ( 1,6 à $2,3 \%$ vs 0,4 


\begin{tabular}{|c|c|c|c|c|c|}
\hline Cohorte & Statut & Nombre d'individus & Détection de iciHHV-6 (\%) & odds ratio [IC] & $\mathbf{P}$ \\
\hline \multirow{2}{*}{ POP } & PE & 249 & $5(2,0)$ & \multirow[b]{2}{*}{-} & \multirow[b]{2}{*}{-} \\
\hline & $T$ & 3598 & $29(0,8)$ & & \\
\hline \multirow{2}{*}{ Case-control } & PE & 218 & $5(2,3)$ & \multirow[b]{2}{*}{-} & \multirow{2}{*}{-} \\
\hline & $\mathrm{T}$ & 256 & $1(0,4)$ & & \\
\hline \multirow{2}{*}{ POP + Case-control } & PE & 467 & $10(2,1)$ & 2,8 & \multirow{2}{*}{0,008} \\
\hline & $\mathrm{T}$ & 3854 & $30(0,8)$ & {$[1,4-5,6]$} & \\
\hline \multirow{2}{*}{$\begin{array}{c}\text { GOPEC } \\
\text { Diverses études sur de grandes } \\
\text { populations de sujets sains }\end{array}$} & PE & 740 & $12(1,6)$ & 2,5 & \multirow{2}{*}{0,001} \\
\hline & $\mathrm{T}$ & 61549 & $403(0,7)$ & {$[1,4-4,4]$} & \\
\hline
\end{tabular}

Tableau II. Détection de iciHHV-6 dans le sang du cordon ombilical dans diverses cohortes d'étude sur la pré-éclampsie (POP, Case-control, GOPEC) et dans le sang périphérique de sujets sains (diverses études sur de grandes populations). POP : pregnancy outcome prediction study (la cohorte POP inclut les mères des enfants de l'étude analysant le transcriptome du placenta); GOPEC: United Kingdom genetics of pre-eclampsia cohort study. PE : pré-éclampsie ; T : témoin ; IC : intervalle de confiance à $95 \%$. Les odds ratios (et leurs intervalles de confiance à $95 \%$ ) ont été calculés en comparant la détection de iciHHV-6 dans les groupes avec pré-éclampsie versus témoins, et sont statistiquement significatifs ( $p \leq 0,001)$.

à $0,8 \%$ ). Les odds ratios indiquaient que le risque de pré-éclampsie chez les mères d'enfant porteur de iciHHV-6 était plus que doublé (Tableau II).

\section{Infection par HHV-6, infertilité et pathologie de la grossesse}

D'autres auteurs ont aussi mis en évidence un lien entre infection par HHV-6 et infertilité ou grossesse pathologique. Chez des femmes ayant une infertilité inexpliquée, I'ADN de HHV-6A a été retrouvé dans $43 \%$ des biopsies d'endomètre, alors qu'il était indétectable dans le groupe témoin [8]. Après avoir isolé les différents types cellulaires de l'endomètre (cellules épithéliales, stromales et mononucléées), la détection par PCR a montré que seules les cellules épithéliales contenaient HHV-6A (en moyenne 4 copies de génome viral par cellule). Malgré cette forte charge virale, l'absence de détection dans d'autres cellules, notamment celles du sang périphérique où seul I'ADN de HHV-6B était détecté, permet d'exclure un iciHHV-6A. II s'agit donc d'une réactivation de HHV6A localisée à l'épithélium endométrial. De plus, l'expression de protéines virales précoces ( $\mathrm{p} 4 \mathrm{l}$ et Iદ2) et tardives (gpll6) variait selon la localisation et les phases du cycle menstruel, suggérant des conditions de réactivation de HHV-6A dépen- dantes de facteurs du micro-environnement tissulaire, notamment hormonaux, comme un taux plus élevé d'œstradiol. L'infection par HHV-6A modifiait l'expression locale des cytokines (taux plus élevés d'interleukine 10 et plus faibles d'interféron $\gamma$, déjà observés dans des études précédentes), ainsi que le phénotype des cellules NK (natural killer) de I'endomètre, avec une augmentation de la réponse spécifique anti-HHV-6A. Dans un modèle in vitro d'implantation de l'embryon, la coculture de trophoblastes sur une monocouche de cellules de l'épithélium endométrial prélevées chez des femmes avec ou sans infection par HHV$6 \mathrm{~A}$, a montré que l'infection limitait l'invasion de la muqueuse utérine par le trophoblaste de l'embryon [9], et diminuait l'expression de molécules de signalisation essentielles à la nidation (HLA-G et MUCl), ce qui pourrait mener à une fausse couche. D'autres équipes ont trouvé des corrélations entre infections par HHV-6, mais aussi par d'autres virus ou herpèsvirus, et diverses complications de la grossesse comme l'hypertension artérielle gravidique, les avortements spontanés ou la naissance prématurée [10-12]. Toutes ces études nécessitent d'être approfondies pour mieux définir le rôle que pourrait jouer la réactivation de souches intégrées ou non de HHV-6, plus particulièrement de l'espèce HHV-6A, dans certaines difficultés de procréation ou complications de la grossesse. $\diamond$ Role of human herpesviruses 6 (HHV-6) in predisposition to pre-eclampsia

\section{LIENS D'INTÉRÊT}

Les auteures déclarent n'avoir aucun lien d'intérêt concernant les données publiées dans cet article.

\section{RÉFÉRENCES}

1. Gautheret-Dejean A. Actualités sur la forme intégrée du génome du sixième herpesvirus humain au génome humain (iciHHV-6). Med Sci (Paris) 2017 ; $33: 730-1$.

2. Bonnafous $P$, Marlet J, Bouvet $D$, et al. Fatal outcome after reactivation of inherited chromosomally integrated HHV-6A (iciHHV-6A) transmitted through liver transplantation. Am J Transplant 2018 ; 18 : 1548-51.

3. Gravel A, Dubuc I, Morissette G, et al. Inherited chromosomally integrated human herpesvirus 6 as a predisposing risk factor for the development of angina pectoris. Proc Natl Acad Sci USA 2015 ; 112 : 8058-63.

4. Hill JA, Magaret AS, Hall-Sedlak R, et al. Outcomes of hematopoietic cell transplantation using donors or recipients with inherited chromosomally integrated HHV-6. Blood 2017 ; 130 : 1062-9.

5. Hall CB, Caserta MT, Schnabel KC, et al. Transplacental congenital human herpesvirus 6 infection caused by maternal chromosomally integrated virus. J Infect Dis $2010 ; 201: 505-7$.

6. Gaccioli F, Lager S, de Goffau MC, et al. Fetal inheritance of chromosomally integrated human herpesvirus 6 predisposes the mother to preeclampsia. Nat Microbiol 2020 ; 5 : 901-8.

7. Huang $Y$, Hidalgo-Bravo $A$, Zhang $\varepsilon$, et al. Human telomeres that carry an integrated copy of human herpesvirus 6 are often short and unstable, facilitating release of the viral genome from the chromosome. Nucleic Acids Res 2014 ; 42 : 315-27. 


\section{RÉFÉRENCES}

8. Marci R, Gentili V, Bortolotti D, et al. Presence of HHV $6 \mathrm{~A}$ in endometrial epithelial cells from women with primary unexplained infertility. PLoS One 2016; 11 : e0158304.

9. Bortolotti D, Gentili V, Rotola A, et al. HHV-6A infection of endometrial epithelial cells affects immune profile and trophoblast invasion. Am J Reprod Immunol 2019; 82 : el3174.

10. Gervasi MT, Romero R, Bracalente G, et al. Viral invasion of the amniotic cavity in the midtrimester of pregnancy. J Matern Fetal Neonatal Med 2012; 25 : 2002-13.

11. Gibson CS, Goldwater P, MacLennan AH, et al. Fetal exposure to herpesviruses may be associated with pregnancy-induced hypertensive disorders and preterm birth in a Caucasian population. BJOG 2008; $115: 492-500$.

12. Drago F, Ciccarese G, Herzum A, et al. Pityriasis rosea during pregnancy: major and minor alarming signs. Dermatology 2018 ; 234 : 31-6.

\section{NOUVELLE}

\section{L'hepcidine}

\section{Fer de lance de la guérison des maladies chroniques inflammatoires de l'intestin}

Cyril Renassia, Carole Peyssonnaux
Institut Cochin, Inserm, CNRS, Université de Paris, Laboratoire d'excellence GR-Ex, 24 rue du Faubourg Saint-Jacques, 75014 Paris, France. carole.peyssonnaux@inserm.fr
> La maladie de Crohn et la rectocolite hémorragique sont les deux principales maladies chroniques inflammatoires de l'intestin ( $\mathrm{MICl})$. Elles se caractérisent par une inflammation sévère de la paroi intestinale, une hyperactivité du système immunitaire digestif, et par des anomalies de la composition du microbiote intestinal. Les $\mathrm{MICl}$ sont le plus souvent diagnostiquées chez les personnes âgées de 20 à 30 ans. Leur incidence a augmenté dans le monde au cours des dernières décennies. On estime que plus d'un million de personnes aux États-Unis et 2,5 millions en Europe en sont atteintes. En outre, elles sont apparues dans les pays nouvellement industrialisés d'Asie, d'Amérique du Sud et du Moyen-Orient, où leur incidence en 2025 pourrait approcher celle des pays d'Europe [1]. Les patients atteints de $\mathrm{MICl}$ présentent un risque accru de cancer colorectal par rapport à la population générale [2]. Ainsi, la découverte d'un traitement efficace des $\mathrm{MICl}$ est un enjeu majeur pour les années à venir, car actuellement, il n'existe aucun traitement agissant sur la cause de ces maladies. La prise en charge thérapeutique actuelle vise seulement à atténuer les symptômes de la maladie, en calmant la douleur, souvent intense, en réduisant l'inflammation et la fatigue, et en facilitant la prise alimentaire.
Les $\mathrm{MICl}$, mais également leurs complications, telles que les cancers colorectaux et les infections gastro-intestinales, peuvent, en provoquant des saignements chroniques de la muqueuse du tube digestif, entraîner une anémie par carence martiale (ou sidéropénie), qui nécessite alors une supplémentation en fer, souvent peu efficace. D'autre part, les saignements entraînent une augmentation du fer dans la lumière intestinale, dont les conséquences sur la composition du microbiote intestinal sont inconnues à ce jour. Le fer est un nutriment essentiel à tout organisme vivant, des micro-organismes jusqu'à l'homme. La virulence des bactéries dépend notamment de leur capacité à assimiler le fer de I'hôte qui les héberge

$\rightarrow$ Voir la Synthèse de S. Vaulont et I. Schalk, $m / s n^{\circ} 8-9$, août-septembre 2015, page 756 [3] $(\rightarrow)$.

Chez l'homme, le fer est indispensable, non seulement pour le métabolisme cellulaire (en tant que cofacteur de réactions enzymatiques, de la réplication de I'ADN, etc.), mais également pour permettre à l'hémoglobine contenue dans les globules rouges de transporter l'oxygène vers les différents tissus. Si le fer vient à manquer, une anémie apparaît, ce qui peut entraîner une oxygénation insuffisante des tissus. En revanche, une surcharge en fer peut induire des dommages cellulaires, en facilitant la production de formes réactives de l'oxygène (ROS) [4]. Les taux de fer doivent donc être finement régulés pour éviter les conséquences d'un déficit ou, au contraire, d'une surcharge toxique pour les cellules. L'hepcidine est l'hormone majeure de l'homéostasie du fer dans l'organisme [5]. II s'agit d'un petit peptide cationique, de 25 acides aminés, principalement produit par le foie en réponse à la surcharge en fer ou à une inflammation. L'hepcidine agit sur la ferroportine, la seule protéine exportatrice de fer connue, en limitant sa capacité à libérer le fer intracellulaire, soit comme un «bouchon » empêchant l'exportation, soit en induisant sa dégradation [6]. La ferroportine est principalement exprimée à la surface des macrophages érythrophagocytaires (cellules qui reconnaissent et éliminent les hématies sénescentes), et à la surface baso-latérale des entérocytes du duodénum, où elle facilite le recyclage du fer des globules rouges sénescents et l'importation de fer alimentaire, respectivement [7]. II est désormais établi que les maladies de surcharge en fer primaires (hémochromatoses) sont associées à un défaut de production d'hepcidine [8]. 